\title{
Genetically Encoded CRISPR components Yield Efficient Gene Editing in the Invasive Pest, Drosophila suzukii
}

\author{
Authors \\ Nikolay P. Kandul ${ }^{1, *}$, Esther J. Belikoff ${ }^{2,}$, Junru Liu ${ }^{1}$, Anna Buchman ${ }^{1}$, Fang Li ${ }^{2}$, Akihiko Yamamoto ${ }^{2}$, Ting \\ Yang $^{1}$, Isaiah Shriner ${ }^{1}$, Maxwell J. Scott ${ }^{2, \dagger}$ and Omar S. Akbari ${ }^{1, \dagger}$ \\ Affiliations \\ ${ }^{1}$ Division of Biological Sciences, Section of Cell and Developmental Biology, University of California, San \\ Diego, La Jolla, CA, 92093. \\ ${ }^{2}$ Department of Entomology and Plant Pathology, North Carolina State University, Raleigh, NC, 27695. \\ "contributed equally \\ †Correspondence to: Maxwell J. Scott (mjscott3@ncsu.edu) and Omar S. Akbari, (oakbari@ucsd.edu)
}

\section{Running title}

Efficient gene editing in Drosophila suzukii

Keywords

CRISPR, Cas9, Drosophila suzukii, Gene editing

\begin{abstract}
Originally from Asia, Drosophila suzukii (Matsumura, 1931, Diptera: Drosophilidae) is presently a global pest of economically important soft-skinned fruits. Also commonly known as spotted wing Drosophila (SWD), it is largely controlled through repeated applications of broad-spectrum insecticides. There is a pressing need for a better understanding of SWD biology and for developing alternative environmentally-friendly methods of control. The RNA-guided Cas9 nuclease has revolutionized functional genomics and is an integral component of several recently developed genetic strategies for population control of insects. Here we have developed transgenic strains that encode three different terminators and four different promoters to express Cas 9 in both the soma and/or germline of SWD. The Cas9 lines were evaluated through genetic crossing to transgenic lines that encode single guide RNAs targeting the conserved X-linked yellow body and white eye genes. We find that several Cas9/gRNA lines display very high editing capacity. Going forward, these tools will be instrumental for evaluating gene function in SWD and may provide tools useful for the development of new genetic strategies for control of this invasive species.
\end{abstract}




\section{Introduction}

Drosophila suzukii, commonly known as Spotted Wing Drosophila (SWD), is a significant crop pest of many soft-skinned fruits that has recently invaded much of the world (1-3). Unlike other fly species that infest overripe, or rotting fruits, SWD targets ripening fruits $(1,3,4)$. SWD females $(P)$ use a serrated ovipositor to pierce the fruit skin, and deposit progeny inside to consume the fruit (1). The external wounds generated by oviposition alone leave the fruit vulnerable to secondary infections caused by pathogens including bacteria, yeasts, and fungi. The short generation time of SWD contributes to its rapid infestation, resulting in significant revenue losses (5-7). Endemic to East Asia, SWD has become established around much of Europe, North America, and South America since 2008 (6-9), with modeling predicting even further spread (10). Given the invasiveness of SWD, and the significant crop damages, there exists a pressing need to generate molecular tools that can be used for both gaining a better understanding of SWD biology and to innovate alternative environmentally-friendly control methodologies.

SWD is largely controlled through the use of insecticides (11-14). However, insecticide applications can provide limited protection, indiscriminately affect beneficial species (15), and insecticide resistance has emerged (16). Environmentally-friendly species-specific methods of insect pest control, such as the sterile insect technique (SIT), and incompatible insect technique (IIT), are being developed for SWD. In SIT applications, flies are mass reared and exposed to ionizing radiation for sterilization. Then, excess sterilized males ( $\left.\widehat{\gamma}^{\prime} \mathrm{s}\right)$ are repeatedly released to

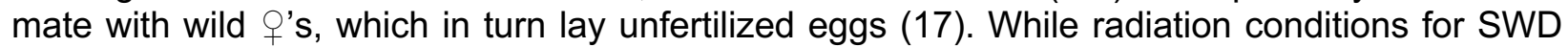
have been identified that produce sterile J's $^{\prime}$ and Q's $(18,19)$, further testing is necessary to

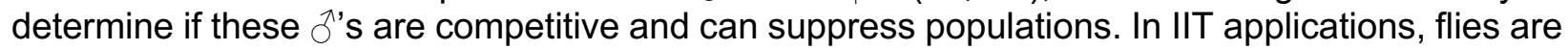
instead infected with the endosymbiont Wolbachia, which has been shown to induce cytoplasmic

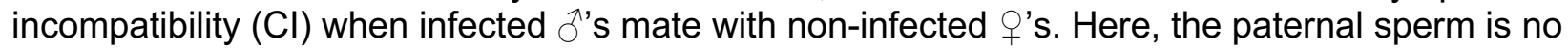
longer recognized by the egg, leaving the egg unfertilized $(20,21)$. While IIT can be a promising application, accidental release of infected $Q$ 's can lead to unintended replacement of a wild population with an infected/resistant one, as $\mathrm{Cl}$ does not occur when infected $O$ 's mate with either

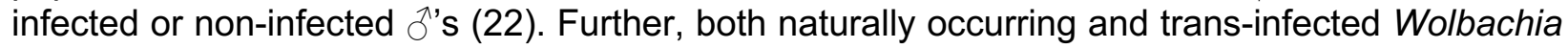
strains do not provide $100 \% \mathrm{Cl}$ in SWD, which is required for an effective IIT (23). The combination of SIT and IIT was recently assessed to improve the sterility of Wolbachia infected SWD. However, the mating competitiveness of released SWD ડ̄'s was not assessed (24). Thus, while progress has been made, it remains to be determined if SIT and/or IIT can provide an alternative economical means for control of SWD.

Over the past several years there has been significant progress in developing molecular genetic tools that can be used for both gaining a better understanding of SWD biology and to innovate alternative environmentally-friendly control methods. For instance, transgenesis has been achieved by several groups (25-28), a high quality reference genome has been assembled (29), and the versatile RNA-guided CRISPR/Cas9 nuclease (clustered regularly interspaced short palindromic repeats/CRISPR-associated sequence 9) has recently been used for gene editing $(30,31)$. For gene editing in SWD, site-specific mutations were initially made through microinjection of embryos with Cas9/gRNA plasmid DNAs (31). The efficiency of mutagenesis was later improved upon by microinjection with recombinant Cas 9 protein and synthetic gRNA rather than plasmid DNAs (30). This editing efficiency could be further improved by generating transgenic strains that encode Cas9/gRNA, as done in D. melanogaster (32-35). Further, development of effective Cas9/gRNA strains may also optimize site-specific transgenesis methods $(36,37)$, and prove useful for generating a variety of genetic control systems in SWD such as precision-guided SIT (pgSIT) $(38,39)$, or possibly even homing based gene drives $(40$, 41) for population suppression. 
Given the many advantages of generating transgenic lines encoding Cas9/gRNAs, here we report the development and evaluation of such strains in SWD. In total we generated 8 homozygous Cas9 expressing lines that were driven by four separate promoters (nos, vasa, BicC, and ubiq) using three different terminators to provide robust expression in both the soma and/or germline. To evaluate the efficacy of these strains, we also generated strains encoding single guide RNAs that target two conserved $\mathrm{X}$-linked recessive genes known to produce visible phenotypes when disrupted including yellow body and white eye genes. By crossing the Cas9 and gRNA strains together, we find that several strains display remarkably high rates of editing capacity. Going forward, these tools will be invaluable for characterization of gene function and may also prove useful for engineering novel control strategies for this invasive crop pest.

\section{Results}

\section{Development of Cas9 and gRNA encoding strains.}

The aim of the study was to develop a versatile toolbox for gene editing and genome engineering in SWD by generating transgenic strains encoding CRISPR components (i.e. Cas9 and gRNAs). To robustly express and import Cas9 into nuclei, we used Streptococcus pyogenes Cas9 (Cas9) with a nuclear localization sequence (NLS) on either the C-terminal end only (Cas9-NLS (32)), or on both terminals (NLS-Cas9-NLS (42)) (Fig. 1A). To drive expression of Cas9, we used $D$. melanogaster promoters expressed in either early germ cells, vasa (vas) (43) or nanos (nos) (44), or in late germ cells, Bicaudal C (BicC) (45), or in both germ and somatic cells, polyubiquitin $63 E$ (ubiq) (46). Using each promoter, we built four piggyBac constructs that express NLS-Cas9-NLS terminated by a p10 3'-UTR derived from the Autographa californica nucleopolyhedrovirus (AcNPV) $(47,48)$ for strong translation of Cas9, and a red (Opie2-dsRed) transgenesis marker (referred as Cas9.R). We also built two alternative piggyBac constructs that contain either the vas or nos promoters driving expression of the Cas9-NLS terminated with vas or nos 3'UTR's from $D$. melanogaster, with a green (ubiq-ZsGreen) transgenesis marker (referred as Cas9.G) (Fig. 1A). In total, six Cas 9 constructs were engineered that were used to generate 8 homozygous transgenic strains (at least one homozygous transgenic strain per construct), two of which were X-linked (Fig. 1B, Table 1).

To genetically encode the gRNAs in SWD, we engineered two separate constructs encoding gRNAs targeting homozygous viable $X$-linked genes with recessive phenotypes: yellow $(y)$ and white $(w)(30,31)$ (Fig. 1C). Each construct encoded one gRNA driven by the $D$. melanogaster small nuclear RNA U6-3 promoter providing constitutive expression (49). The gRNA $A^{y}$ construct harbors two green transgenesis markers (Opie2-eGFP and 3xP3-eGFP), and the gRNA construct contains one red marker (ubiq-dsRed). Both gRNA ${ }^{y}$ and gRNA ${ }^{\mathrm{w}}$ constructs were assembled in piggyBac plasmids (Fig. 1C), and homozygous $g R N A^{y}$ and $g R N A^{w}$ lines were generated (Fig. 1D, Table 1).

\section{CRISPR-mediated mosaicism in $F_{1}$ progeny}

To assess the functionally of the strains produced, we genetically crossed homozygous Cas 9 's to homozygous gRNA ડ̄'s and examined expected eye and/or body coloration phenotypes in the resulting $\mathrm{F}_{1}$ progeny (Fig. 2A-F; Table S1, S2). This cross was performed to explore the rates of mutagenesis in the $F_{1}$ somatic tissues, which is augmented by maternal deposition of Cas9 (45). High percentages $(61.1 \%-100 \%)$ of $\mathrm{F}_{1}$ trans-heterozygous progeny generated by Cas $9 . R$ q's crossed to $g R N A^{y}$ วิ's had visible yellow, instead of brown, body coloration indicating robust somatic yellow gene disruption ( $y$ - phenotype) (Fig. 2B,C, Table S1). Notably, when the Cas9.R f's were crossed to $g R N A^{w}$ '̄'s (Fig. 2D), we observed much higher variability $(0 \%-100 \%)$ for somatic white disruption in resulting $F_{1}$ progeny (Table S2). While both sexes were affected, the 
majority of $F_{1}$ trans-heterozygous progeny manifested highly variable mosaic eye coloration $(m W)$, and a smaller fraction of $F_{1}$ progeny had complete white eye color $(w-$, Fig. $2 E$, $\mathbf{F})$. These results suggest that some somatic cells in mosaic flies harbored at least one wildtype white (wt $\left.w^{+}\right)$allele, or possibly a functional resistant $\left(w^{R 1}\right)$ allele. Interestingly, the extensive somatic disruption of yellow and white was induced by all Cas9.R lines, while Cas9.G crossed to $g R N A^{y}$ and $g R N A^{w}$ strains did not appear to induce somatic mutations in the resulting $\mathrm{F}_{1}$ progeny (Tables S1, S2).

\section{Maternal deposition and $F_{1}$ Mosaicism}

To determine whether maternal Cas 9 deposition is essential to achieve high rates of $F_{1}$ mutagenesis, we compared the rates of white mosaicism in $F_{1}$ trans-heterozygous progeny inheriting Cas9 either maternally or paternally (Fig. 2G,H). We tested two Cas9 strains including BicC-Cas9. $R$ and ubiqCas9. $R$ which gave high rates of white mosaicism when Cas 9 was provided maternally. The $\mathrm{F}_{1}$ BicC-Cas9.R/+; gRNA ${ }^{\mathrm{w}} /+$ with maternal Cas 9 showed significantly higher rates of white mosaicism then those with paternal Cas9 $(68.5 \pm 12.6 \%$ vs $25.1 \pm 4.3 \%$ of $w-\& m W$, respectively; $p<0.0002 t$-test with equal variance, Fig. $2 \mathrm{H}$, Table S3). Comparatively, the ubiqCas9.R/+; gRNAw/+ with maternal Cas9 showed significantly lower rates of white mosaicism then those with paternal Cas9 $(78.5 \pm 21.4 \%$ vs $99.7 \pm 0.9 \%$ of $w-\& m W$, respectively, $p<0.035 t$ test with unequal variance, Fig. $2 \mathrm{H}$, Table S3). Taken together, these data indicate that regardless of whether Cas9 was inherited maternally or paternally, high rates of mosaicism were observed in $\mathrm{F}_{1}$ trans-heterozygous progeny.

\section{Cas9/gRNA-mediated heritable mutations}

To estimate the frequency of heritable germline mutations we mated $F_{1}$ trans-heterozygous ' $^{\prime} s$

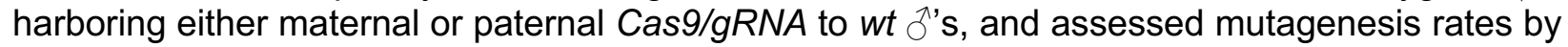
scoring phenotypes in hemizygous $\mathrm{F}_{2}$ गे's progeny (Fig 3A). Both yellow and white are X-linked, therefore $F_{2}$ ठ 's generated from $F_{1}$ trans-heterozygous ${ }^{\prime}$ 's inherit their mothers' $X$ chromosome. If the $F_{1}$ O's encode germline mutations in either yellow or white, then $F_{2}$ ठ's will inherit those $X-$ linked mutations and display visible phenotypes (i.e. non-mosaic complete white eyes or yellow body). Of note, we specifically scored complete yellow and white phenotypes in $\mathrm{F}_{2} \overbrace{}^{\Uparrow}$ progeny here to differentiate heritable germline mutations from somatic mosaic mutations resulting from either maternal deposition or zygotic expression of transgenes. In combination with $g R N A^{y}$, the Cas9.R strains induced significantly higher rates of $y$-alleles than those induced by the Cas9.G strains $(77.6 \pm 12.5 \%$ and $2.0 \pm 2.1 \%$, respectively; $p<0.0001 t$-test with unequal variance, Fig. 3B,C, Table S1). The $\mathrm{F}_{1}$ ubiqCas9.R/+; gRNA $A^{y}++$ 's harboring maternal Cas 9 induced significantly higher levels of $y$ - alleles than those carrying paternal Cas $9(95.4 \pm 4.7 \%$ and $78.5 \pm 1.4$, respectively; $p<0.0003 t$-test with equal variance), while the opposite was observed for $\mathrm{F}_{1}$ vasCas9. $\mathrm{G} \# 6 /+; g R N A^{y /+}+q(0.6 \pm 1.6 \%$ and $3.2 \pm 1.8$, respectively; $p<0.0163 t$-test with equal variance, Fig. 3B). Interestingly, we observed higher variability of heritable $w$ - mutations among replicates for the majority of Cas9 strains (Fig. 3D). For example, independent groups of $\mathrm{F}_{1} \mathrm{BicC}$ -

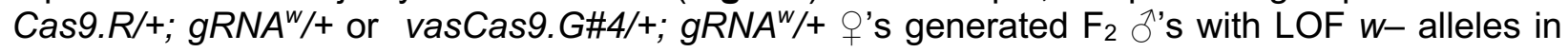
$92.7 \%$ and $1.1 \%$ of progenies, or those with $w-$ in $83.5 \%$ and $0 \%$, respectively (Fig. 3D, Table S2). Note that we did not find the mosaic eye coloration ( $m W$ phenotype, Fig. $2 \mathrm{~F}$ ) in $\mathrm{F}_{2} \sigma^{\lambda}$, they had either $w$ - or $w+$ eye phenotype indicating the absence of somatic mosaicism (Fig. 3E).

\section{Functional resistant alleles at the white locus}

We observed higher variations in both $F_{1}$ and $F_{2}$ mutagenesis of white than compared to yellow (Figs. 2B,E and 3B,D). We hypothesized that the $\mathrm{F}_{2} w+\hat{\jmath}$ may harbor either uncut $w t w+$ alleles or functional repaired alleles that were resistant to Cas $9 / g R N A^{w}$ cleavage (termed $w^{R 1}$ alleles) (Fig. 4A). To explore this hypothesis, we genotyped $\mathrm{F}_{2} w+\lambda$. Interestingly, we did not identify $w t$ $w+$ alleles among $37 \mathrm{~F}_{2} \widehat{o}$ generated from multiple independent crosses. Instead, each genotyped 
$\mathrm{F}_{2}{ }^{\top}$ harbored either a one-base substitution (1bpSUB) or a twelve-base deletion (12bp $\Delta$ ) directly at the white target sequence (Fig. 4B). Both in-frame $w^{R 1}$ alleles preserved $w t$ function of the white gene. The A-to-G substitution in the $1 \mathrm{bpSUB}$ allele did not change the amino acid sequence (i.e. silent mutation), while the $12 \mathrm{bp} \Delta$ allele contains a deletion of four amino acids (Fig. 4B). Notably, the $12 \mathrm{bp} \Delta$ allele was sampled more frequently than the $1 \mathrm{bpSUB}$ allele, though both $w^{R 1}$ alleles were found in crosses with each Cas9.R strain. We also genotyped a few $F_{2} W-\widehat{O}$ and identified diverse LOF resistant alleles (termed $w^{R 2}$ ) induced by Cas $9 / g R N{ }^{w}$ (Fig. 4C). Each genotyped $F_{2} w-\sigma^{\lambda}$ harbored one LOF $w^{R 1}$ allele. Notably, we identified two in-frame LOF deletion alleles $\left(3 \mathrm{bp} \Delta\right.$ and $\left.12 \mathrm{bp} \Delta^{*}\right)$ that could not rescue the wt white function.

\section{Mutant $y$ - strains were established}

Both yellow and white genes have many features that makes them attractive targets and/or tools for genetic research. Therefore, we attempted to establish $w$ - and $y$ - strains to facilitate future genetic research in SWD. To this end, we generated independent $F_{2}$ progeny (Fig. 3), and intercrossed individual $\mathrm{F}_{2}+$ and $\delta$ harboring $y$ - or $w$ - mutant alleles in the absence of both Cas 9 and gRNA transgenes. We were able to establish and maintain eight homozygous $y$ - strains, however we could not establish $w$ - stocks as loss of function $w-\widehat{\sigma}$ were sterile $(30,31)$. We genotyped each $y$ - strain and identified five different insertions/deletions (indel) mutations at the yellow target sequence (Fig. 5). One six-base-deletion (6bp $\Delta$ ) was induced independently in four yellow knockout strains (Fig. 5).

\section{Discussion}

Here we describe the generation and assessment of an array of SWD transgenic strains expressing Cas 9 driven under four promoters from $D$. melanogaster. To evaluate these Cas 9 strains, two gRNA strains targeting X-linked recessive genes were also established. Using Cas 9 and $g R N A$ strains, we demonstrate that the Cas9.R strains result in high rates of mutagenesis of targeted genes, while the Cas9.G strains had more limited efficacy.

Our results indicate that the Cas9.R constructs containing NLS-Cas9-NLS terminated with the p10 3'UTR resulted in the significantly stronger disruption of yellow in both somatic and germ cells than that by the Cas9.G harboring Cas9-NLS and terminated with the vas or nos 3'UTR. Due to random genomic integration of constructs, direct comparison of Cas9 strains is confounded. Nevertheless, the observed differences were consistent among each Cas9.R and Cas9.G strain. The p10 3'-UTR from the Autographa californica nucleopolyhedrovirus (AcNPV) is known to increase efficiency of both polyadenylation and mRNA translation $(47,48)$. In addition, a sevenamino-acid-long SV40 NLS is shared between both Cas9.R and Cas9.G types, while Cas9.R constructs also contain a sixteen-amino-acid bipartite nucleoplasmin NLS (Fig. 1A). The inclusion of an additional NLS into Cas9 may provide increased nuclear localization. The vas and nos 3'UTRs used in the Cas9.G gene constructs would be expected to lead to localization of the Cas9 mRNA to the posterior end of the oocyte (50). In contrast, the p10 3'UTR is not predicted to result in such localization during oogenesis. Thus, our expectation was that the Cas9.G lines would be mostly active in germ cells that develop at the posterior end of the embryo, while the Cas9.R lines would have high activity in both somatic and germ cells. Several generated Cas9.G strains harboring independent insertions of vasCas9.G or nos. Cas9. G did not induce visible $\mathrm{F}_{1}$ somatic mutagenesis of yellow or white in the presence of the corresponding gRNA. Instead they caused the yellow or white mutagenesis in the $\mathrm{F}_{2}$ progeny indicating a possible germline-restricted Cas 9 expression pattern.

We observed a high inter-replicate variability in frequencies of the white disruption in both somatic and germ cells. To explore cause of this variability, we genotyped many $F_{2} w+\widehat{\delta}$ 's and found that each genotyped $w+\delta^{\lambda}$ harbored one of the two functional $w^{R 1}$ alleles. Our results indicate that the Cas9/gRNA ${ }^{\mathrm{w}}$ cleavage of white was efficient. We did not sample wt $w+$ alleles in $\mathrm{F}_{2} \hat{\partial}$, instead 
they each harbored $w^{R 1}$ and $w^{R 2}$ resistant alleles. Both identified $w^{R 1}$ alleles, $1 \mathrm{bpSUB}$ and $12 \mathrm{bp} \Delta$, may have been induced by Cas $9 / \mathrm{gRNA}{ }^{\mathrm{w}}$ or may have existed in the strains. The fact that the same two $w^{R 1}$ alleles were sampled in each cross with four Cas9.R strains also cannot rule out that at least one of them occurred naturally or was generated. However, the $12 \mathrm{bp} \Delta$ allele, which removes 4 amino acids, potentially affects the fitness of its carriers, while the silent substitution is expected to be fitness neutral. The removal of 12 bases at the gRNA target site likely made the $12 \mathrm{bp} \Delta$ allele much more resistant to the further Cas9/gRNA ${ }^{\mathrm{w}}$-mediated cleavage than a single base substitution would; and the $12 \mathrm{bp} \Delta$ allele was sampled more frequently than the $1 \mathrm{bpSUB}$ allele. Therefore, our data suggest that the $1 \mathrm{bpSUB}$ allele could pre-exist in SWD genetic background and might not be completely resistant to the Cas $9 / g R N A^{w}$, while the $12 \mathrm{bp} \Delta$ allele was induced by Cas $9 / g R N A^{w}$ in our experiments.

Over a century ago, the establishment of $D$. melanogaster knockout strains of white and yellow provided valuable genetic background for seminal transgenic research (51). Previous studies demonstrated that unlike $D$. melanogaster, SWD to harboring LOF $w$-alleles were sterile (30, 31), thus preventing the maintenance of the SWD $w$ - strain. Here we describe the development of eight viable SWD $y$ - strains. Five unique $y$ - alleles were characterized from eight $y$ - strains (Fig. 5). These strains may be useful reagents for applications in which fluorescent markers of transgenesis cannot be used, for example when genes of interest (GOI) themselves are linked to fluorescent tags. Therefore, the generated $y$-strains are valuable resources for genetic studies of SWD.

SWD is an invasive species and a close relative of the $D$. melanogaster, the classic genetic model organism for which a vast amount of detailed biological knowledge and a diverse array of genetic tools are available. Given the close phylogenetic proximity, the majority of know-how and genetic tools are easily portable across both Drosophila species and can be applied for the development of genetic methods for effective population control of SWD. The CRISPR/Cas9 technology has been used extensively for the precise genome editing in diverse animal and plant species. Both non-localized and localized methods for insect population control have been developed for $D$. melanogaster using the CRISPR/Cas9 technology $(38,40,52,53)$. The SWD tools strains described here should facilitate precise genetic engineering in the invasive pest species and may provide useful genetic reagents required for the development of genetic population control systems for SWD.

\section{Experimental Procedures}

\section{Molecular Construct design and assembly}

We used the previously described piggyBac plasmids harboring coding sequences of the Cas9T2A-eGFP under different promoters and the Opie2-dsRed marker $(38,42,45)$ to generate SWD Cas9.R strains: vasCas9.R (874Z plasmid, addgene \#112687), BicC.Cas9.R (874R plasmid, addgene \#168295), UbiqCas9.R (874W plasmid, addgene \#112686), and nosCas9.R (874Z1 plasmid, addgene \#112685). The Gibson enzymatic assembly method (54) or standard recombinant DNA methods were used to build the piggyBac transformation plasmids that carried vasCas9.G (addgene \#169012), nosCas9.G (addgene \#169011), gRNA ${ }^{y}$ (982G.2 plasmid, addgene \#168294), and gRNAw (addgene \#169010) constructs. To assemble vasCas9.G and nosCas9. $G$ driven by the $D$. melanogaster vasa and nos promoters, the vasa5'-Cas9-vasa3' and nos5'-Cas9-nos3' fragments were excised from vasa-Cas9 plasmid DNA (Drosophila Genomics Resource Center \#1340 (55)) and pBFv-nosp-Cas9 plasmid (32) (NIG-Fly, Japan), respectively, and then ligated into a piggyBac vector that was cut with $\mathrm{Hpal}$ and PspOMI. The piggyBac vector contains a ZsGreen fluorescent protein marker expressed by the $D$. melanogaster polyubiquitin gene promoter (ubiq-ZsGreen). This was made by excision of eGFP from MS1419 (56) and replacement with ZsGreen from pB[Lchsp83-ZsGreen] (57). We utilized previously described 
yellow (45) and white (31) gRNA sequences to build the gRNA and $g R N A^{w}$ piggyBac plasmids (Fig. 1C). To assemble $g R N A^{y}$, the yellow gRNA targeting an identical sequence in $D$. melanogaster ${ }^{(45)}$ and SWD yellow exon\#2 (DS10_00005318 (58)) was encoded into overlapping primers that amplified the D. melanogaster U6-3 promoter on one side and the single chimeric gRNA scaffold $(49,59)$ with the poly $(T)_{6}$ termination on the other side as previously described (38), and cloned into a piggyBac plasmid habording the Opie2-eGFP-SV40 marker. Then, the additional 3xP3-eGFP-SV40 marker was added upstream from U6-3-gRNA ${ }^{y}$. To build $g R N A^{w}$, the U6:3-ex2 plasmid that contains white gRNA targeting SWD white exon\#2 (DS10_00006062 (58)) and $D$. melanogaster U6:3 promoter and terminator (31) was digested with BgllI and the excised U6:3p-gRNA-U6:3t fragment ligated with the piggyBac vector MS1425-p10 that contains a ubiqdsRed marker. MS1425-p10 was derived from MS1425 (56) by excision of the SV40 pA sequence and replacement with $\mathrm{p} 10 \mathrm{pA}$.

\section{SWD rearing and transgenesis}

SWD were maintained on a cornmeal-yeast-agar diet at $21^{\circ} \mathrm{C}$ with a $12 / 12 \mathrm{~h} \mathrm{light/dark}$ cycle at UCSD and/or NCSU. All test crosses were performed under these conditions except for those with the vasCas9.G and nosCas9.G lines and the U6:3-ex2 white gRNA lines that were performed at $25^{\circ} \mathrm{C}$. In both facilities, SWD flies were kept in an institutional biosafety committeeapproved ACL1 (NCSU) and ACL2 (UCSD) insectaries and handled by limited expert investigators to prevent any unintended release of SWD. The SWD wildtype (wt) strains used in the study originated from Corvallis, OR (25) and North Carolina (31). Embryo injections were carried out at Rainbow Transgenic Flies, Inc. (http://www.rainbowgene.com) or at the NC State University insect transgenesis facility. Plasmids diluted in water to 200-300 $\mu \mathrm{g} / \mu \mathrm{l}$ were injected into freshly collected embryos of the SWD harboring Hsp70Bb-piggyBacTransposase (26) or the North Carolina wild type strain with piggyBac helper plasmid(60). $\mathrm{G}_{0}$ adults emerging from injected embryos were outcrossed to SWD wt flies, and their $\mathrm{G}_{1}$ progeny were screened for the expression of specific fluorescent markers with the Leica M165FC fluorescent stereomicroscope.

\section{SWD genetics}

To establish stable SWD transgenic lines that can be maintained over multiple generations, SWD expressing an independent insertion of a particular construct were repeatedly inter-crossed over multiple generations to generate a homozygous stock. The homozygosity of generated stocks was confirmed by test crosses using homozygous transgenic lines crossed to wt flies and scoring specific transgenic markers in their progeny. To our surprise, we observed that freshly eclosed SWD $q$ would frequently mate with older $\delta$; and, unlike $D$. melanogaster, the freshly eclosed phenotype by itself is not a sufficient indicator of the $q$ virginity. Therefore, to ensure that only virgin $q$ were used for genetic crosses, vials with eclosing flies were cleared up multiple times daily to remove old $\hat{\sigma}$.

We set the majority of genetic crosses in one direction, Cas 9 virgins $q$ were mated to $g R N A \nrightarrow$, to stimulate the gene disruption in somatic tissues of the $F_{1}$ progeny by maternal Cas 9 deposition $(38)^{(45)}$. Seven to ten homozygous Cas 9 virgin $q$ were crossed to $7-10$ homozygous gRNA ${ }^{\lambda}$ in each vial, and the somatic disruption of yellow or white was scored in the $F_{1}$ progeny (Fig. 2AF). In addition to the complete absence of eye pigmentation (white eyes, $w$ - phenotype), mosaic eye coloration was frequently observed in $\mathrm{F}_{1}$ trans-heterozygous Cas $9 . R /+; g R N A^{w} /+$ flies of both sexes: it was recorded as a $m W$ phenotype. We also performed genetic crosses with the paternal Cas9 for BicC.Cas9.R and ubiqCas9.R to assess the role of maternal Cas 9 carryover on somatic gene disruption and to ascertain the insertion of $u$ biq Cas9.R on the $X$ chromosome, respectively 
(Fig. 2G-H). Both yellow and white genes are located on the X chromosome; and SWD 's's have only one $X$ chromosome (i.e. hemizygous), which they inherit from their mothers. Therefore, to assess the mutation frequency in germ cells, $7-10 \mathrm{~F}_{1}$ trans-heterozygous virgin $q$ were crossed to $10 w t \hat{\delta}$, and $y$ - or $w$ - phenotype was scored in the $F_{2} \hat{o}$ progeny. Flies were scored and imaged on the Leica M165FC fluorescent stereomicroscope equipped with the Leica DMC2900, View4K or Leica DFC500 camera.

\section{Genotyping the yellow and white target loci}

To explore the molecular changes that caused resistant (R2) and functional in-frame resistant (R1) alleles. We PCR amplified a genomic region containing the target site for gRNA ${ }^{y}$ or gRNA ${ }^{\mathrm{w}}$ (Fig. 1C) using single-fly genomic DNA preps (38)(45) from individual $F_{2} \delta$, which were scored for yellow or white phenotype. The 397bp PCR fragment of yellow target (exon \#2) was amplified with 5'-GAATTCCAGCCACTCTGACTTATATCAATATGG-3' (982G.s10F) and 5'CAGGAGTAGGCAATTAAACCATAGCCC-3' (982G.s11R); and 5'GTGCCAGCACACGATCATCGGAGTGC-3' (982A.s11R) and 5'TGAGAAGAAGTCGACGGCTTCGCTGG-3' (982A.s10F) primers were used to amplify the 322bp PCR fragment of white target (exon \#2). PCR amplicons were purified using QIAquick PCR purification kit (QIAGEN), and sequenced in both directions with Sanger method at Genewiz, Inc. To characterize molecular changes at the targeted sites, sequence AB1 files were aligned against the corresponding reference sequences in SnapGene 4.

\section{Statistical analysis}

Statistical analysis was performed in JMP8.0.2 by SAS Institute Inc. At least three biological replicates were used to generate statistical means for comparisons. $P$ values were calculated for a two-sample Student's t-test with equal or unequal variance. O'Brien's test was used to assess that the variance is equal. All plots were constructed using Prism9 for macOS by GraphPad Software, LLC.

\section{Acknowledgments}

We thank Amarish Yadav for molecular analysis of the yellow mutant lines. This work was supported in part by funding from Agragene Inc., California Cherry Board, and the Washington Tree Fruit Research Commission awarded to O.S.A. and from the National Institute of Food and Agriculture, U.S. Department of Agriculture Specialty Crops Research Initiative under agreement No. 2015-51181-24252 awarded to M.J.S. The views, opinions, and/or findings expressed are those of the authors and should not be interpreted as representing the official views or policies of the U.S. government.

\section{Ethical conduct of research}

We have complied with all relevant ethical regulations for animal testing and research and conformed to the UCSD institutionally approved biological use authorization protocol (BUA \#R2401).

\section{Author Contributions}

O.S.A and M.J.S. conceived the study. N.P.K., E.J.B, F.L., A.Y., M.J.S. and A.B. designed experiments. N.P.K., EJ.B., A.Y. and A.B. obtained genetic cross data. A.B., T.Y., F.L. and I.S. 
designed and assembled constructs, A.B., F.L., and I.S. made transgenic lines, N.P.K. and J.L. performed molecular analyses. All authors approved the final manuscript.

\section{Disclosures}

O.S.A is a founder of Agragene, Inc., has an equity interest, and serves on the company's Scientific Advisory Board. The terms of this arrangement have been reviewed and approved by the University of California, San Diego in accordance with its conflict of interest policies. All other authors declare no competing interests.

\section{Data availability}

Complete maps and Plasmid DNA assembled in the study were deposited at Addgene.org and available for distribution (Table 1). The SWD strains generated here will be made available upon request. 
Table 1. SWD transgenic strains generated in the study.

\begin{tabular}{|c|c|c|c|c|c|}
\hline Strain & Construct ID & $\begin{array}{l}\text { Strain } \\
\text { marker }\end{array}$ & $\begin{array}{l}\text { Sequence } \\
\text { reference }\end{array}$ & $\begin{array}{c}\text { Strain } \\
\text { availability }\end{array}$ & $\begin{array}{c}\text { Additional } \\
\text { information }\end{array}$ \\
\hline vasCas9.R & $\begin{array}{l}\text { vasCas9.R } \\
(874 Z)\end{array}$ & $\begin{array}{l}\text { Opie2- } \\
\text { dsRed }\end{array}$ & $\begin{array}{l}\text { AddGene } \\
\# 112687\end{array}$ & $\begin{array}{l}\text { O. Akbari at } \\
\text { UCSD }\end{array}$ & $\begin{array}{l}\text { Cas9 expressed in } \\
\text { somatic and germ } \\
\text { cells }\end{array}$ \\
\hline BicC.Cas9.R & $\begin{array}{l}\text { BicC.Cas9.R } \\
\quad(874 \mathrm{R})\end{array}$ & $\begin{array}{l}\text { Opie2- } \\
\text { dsRed }\end{array}$ & $\begin{array}{l}\text { AddGene } \\
\# 168295\end{array}$ & $\begin{array}{l}\text { O. Akbari at } \\
\text { UCSD }\end{array}$ & $\begin{array}{l}\text { Cas9 expressed in } \\
\text { late germ cells }\end{array}$ \\
\hline ubiqCas9.R & $\begin{array}{l}\text { ubiqCas9.R } \\
(874 \mathrm{~W})\end{array}$ & $\begin{array}{l}\text { Opie2- } \\
\text { dsRed }\end{array}$ & $\begin{array}{l}\text { AddGene } \\
\# 112686\end{array}$ & $\begin{array}{l}\text { O. Akbari at } \\
\text { UCSD }\end{array}$ & $\begin{array}{l}\text { X-linked Cas9 } \\
\text { expressed in somatic } \\
\text { and germ cells }\end{array}$ \\
\hline nosCas9.R & $\begin{array}{c}\operatorname{nosCas9.R} \\
(874 Z 1)\end{array}$ & $\begin{array}{l}\text { Opie2- } \\
\text { dsRed }\end{array}$ & $\begin{array}{l}\text { AddGene } \\
\# 112685\end{array}$ & $\begin{array}{l}\text { O. Akbari at } \\
\text { UCSD }\end{array}$ & $\begin{array}{c}\text { Cas9 expressed in } \\
\text { somatic and germ } \\
\text { cells }\end{array}$ \\
\hline vasCas9.G\#4 & vasCas9.G & $\begin{array}{c}\text { ubiq- } \\
\text { ZsGreen }\end{array}$ & $\begin{array}{c}\text { Addgene\# } \\
169012\end{array}$ & $\begin{array}{l}\text { M. Scott at } \\
\text { NCSU }\end{array}$ & $\begin{array}{l}\text { Cas9 expressed in } \\
\text { germ cells }\end{array}$ \\
\hline vasCas9.G\#6 & vasCas9.G & $\begin{array}{l}\text { ubiq- } \\
\text { ZsGreen }\end{array}$ & $\begin{array}{c}\text { Addgene\# } \\
169012\end{array}$ & $\begin{array}{l}\text { M. Scott at } \\
\text { NCSU }\end{array}$ & $\begin{array}{l}\text { Cas9 expressed in } \\
\text { germ cells }\end{array}$ \\
\hline nosCas9.G\#14 & nosCas9.G & $\begin{array}{c}\text { ubiq- } \\
\text { ZsGreen }\end{array}$ & $\begin{array}{c}\text { Addgene\# } \\
169011\end{array}$ & $\begin{array}{l}\text { M. Scott at } \\
\text { NCSU }\end{array}$ & $\begin{array}{l}\text { Cas } 9 \text { expressed in } \\
\text { germ cells }\end{array}$ \\
\hline nosCas9.G\#36 & nosCas9.G & $\begin{array}{c}\text { ubiq- } \\
\text { ZsGreen }\end{array}$ & $\begin{array}{c}\text { Addgene\# } \\
169011\end{array}$ & $\begin{array}{l}\text { M. Scott at } \\
\text { NCSU }\end{array}$ & $\begin{array}{c}\text { X-linked Cas9 } \\
\text { expressed in germ } \\
\text { cells }\end{array}$ \\
\hline gRNA $^{y}$ & $\begin{array}{l}\text { gRNAy }^{y} \\
(982 G .2)\end{array}$ & $\begin{array}{l}\text { Opie2- } \\
\text { eGFP, } \\
\text { 3xP3-eGFP }\end{array}$ & $\begin{array}{l}\text { AddGene } \\
\# 168294\end{array}$ & $\begin{array}{l}\text { O. Akbari at } \\
\text { UCSD }\end{array}$ & $\begin{array}{c}\text { gRNA targets yellow } \\
\text { exon \#2 }\end{array}$ \\
\hline gRNAw & gRNA $^{w}$ & ubiq-dsRed & $\begin{array}{c}\text { Addgene\# } \\
169010\end{array}$ & $\begin{array}{l}\text { M. Scott at } \\
\text { NCSU }\end{array}$ & $\begin{array}{c}\text { gRNA targets white } \\
\text { exon \#2 }\end{array}$ \\
\hline
\end{tabular}



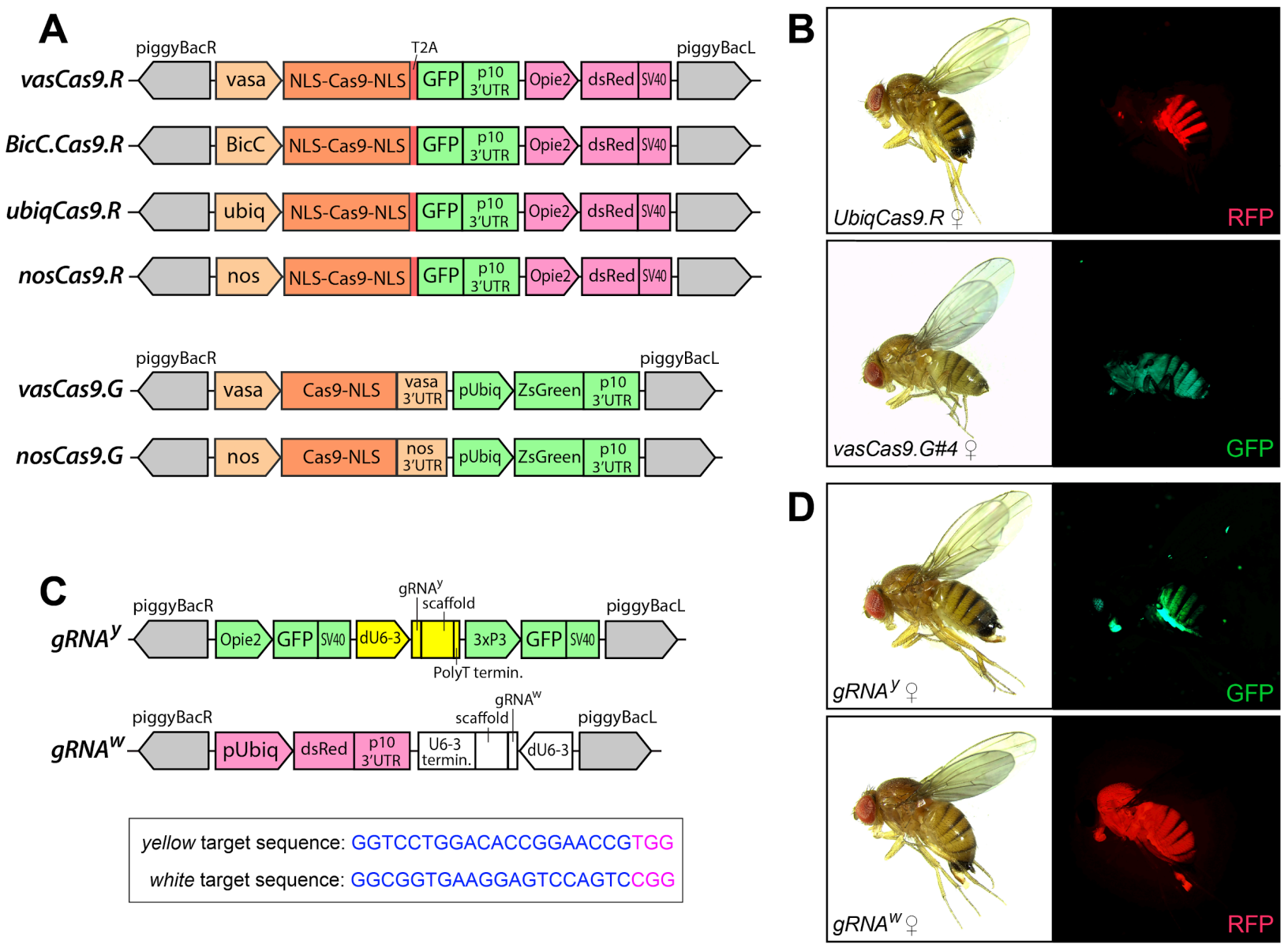

Figure 1. Schematic maps of genetic constructs and images of transgenic SWD. (A) Schematic maps of two sets of Cas9 constructs. The first four Cas9 constructs harbor a human-codon-optimized SpCas9 (Cas9) (61) coding sequence (CDS) surrounded by two nuclear localization sequences (NLS-Cas9-NLS), linked to the eGFP CDS at its C-end via a self-cleaving T2A sequence, and terminated by the p10 3'-UTR from the Autographa californica nucleopolyhedrovirus (AcNPV) (48). The SpCas9 is expressed in early germ cells under vasa (vas) and nanos (nos) promoters, in late germ cells with Bicaudal C (BicC), and in both germ and somatic cells with Ubiquitin 63E (ubiq) promoter. These constructs also contain a red transgenesis marker (Opie2-dsRed). The second group of Cas9 constructs carry a human-codon-optimized SpCas9 expressed under the vas or nos promoter, and terminated with a single NLS (Cas9-NLS) and the corresponding vas and nos 3'UTR, as well as a green transgenesis marker (Ubiq-ZsGreen). (B) Images of homozygous transgenic SWD Cas9 o flies generated with UbiqCas9.R and VasCas9.G. (C) Schematic maps of two gRNA constructs, and the targeted sequences in both yellow and white loci. The gRNA construct harbors the yellow gRNA (gRNA ${ }^{y}$ ) with a scaffold expressed with the Dmel pU6-3 promoter and terminated by a PolyT terminator, and two green transgenesis markers, Opie2-GFP and 3xP3-GFP. The gRNAw construct harbors the white gRNA (gRNAw) with a scaffold expressed with the same Dmel pU6-3 promoter and terminated the pU6-3 terminator sequence, and a red transgenesis marker, Ubiq-dsRed. (D) Images of homozygous SWD $g R N A^{y}$ and $g R N A^{w}$. . Both sets of RGB images for each $q$ fly were taken under the white light and corresponding fluorescent light illumination. 

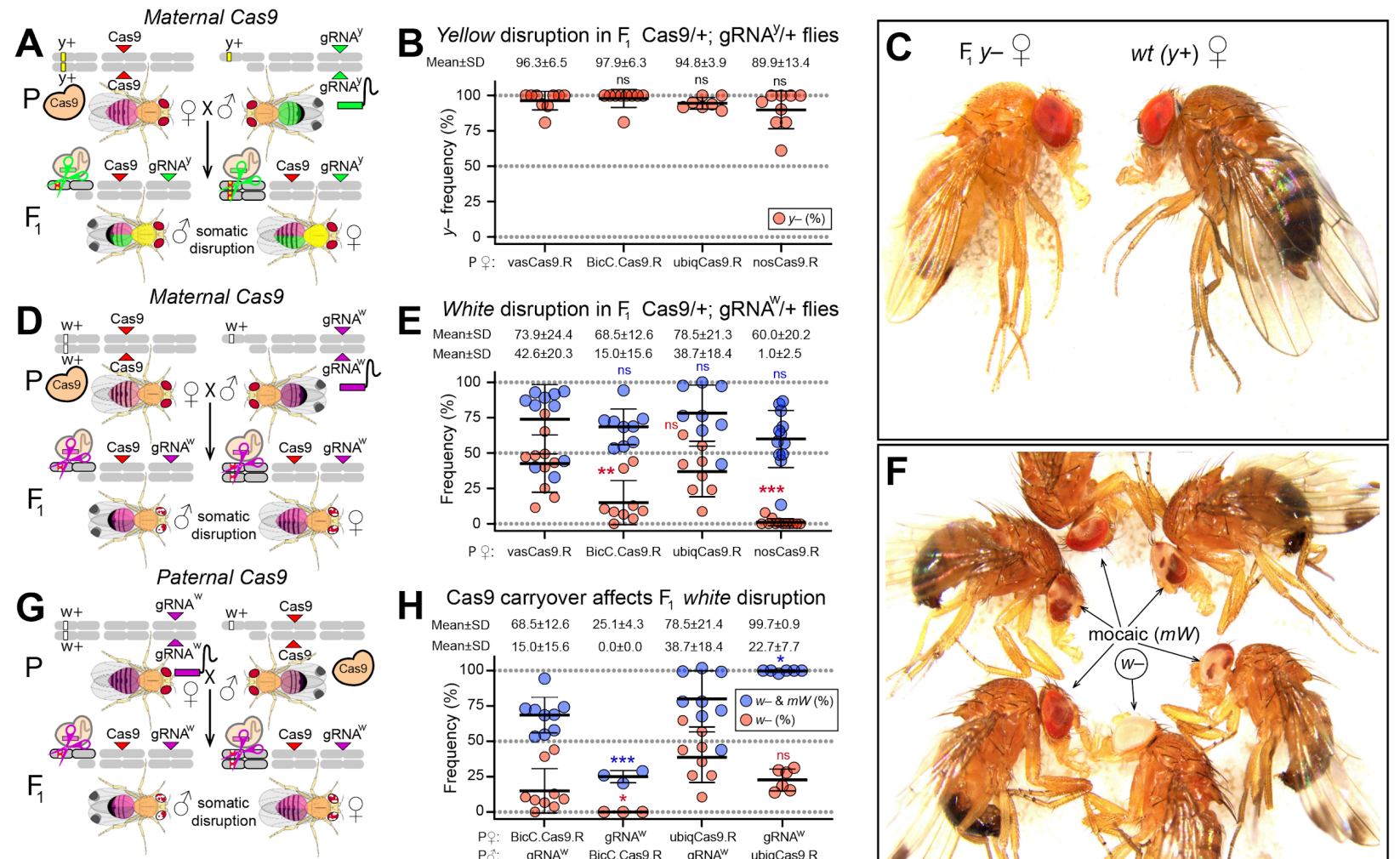

E White disruption in $\mathrm{F}_{1} \mathrm{Cas} 9 /+; \mathrm{gRNA}^{\mathrm{W}} /+$ flies $\begin{array}{lllll}\text { MeantSD } & 73.9 \pm 24.4 & 68.5 \pm 12.6 & 78.5 \pm 21.3 & 60.0 \pm 20.2 \\ \text { MeantSD } & 42.6 \pm 20.3 & 15.0+15.6 & 38.7 \pm 18.4 & 10+2.5\end{array}$ MeantSD $\quad 42.6 \pm 20.3 \quad 15.0 \pm 15.6 \quad 38.7 \pm 18.4 \quad 1.0 \pm 2.5$

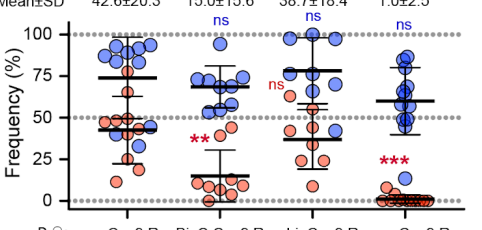

P P: vasCas9.R Bicc.Cas9.R ubiqCas9.R nosCas9.R

H Cas9 carryover affects $F_{1}$ white disruption $\begin{array}{lllll}\text { MeantSD } & 68.5 \pm 12.6 & 25.1 \pm 4.3 & 78.5 \pm 21.4 & 99.7 \pm 0.9\end{array}$ MeantSD $\quad 15.0 \pm 15.6 \quad 0.0 \pm 0.0 \quad 38.7 \pm 18.4 \quad 22.7 \pm 7.7$
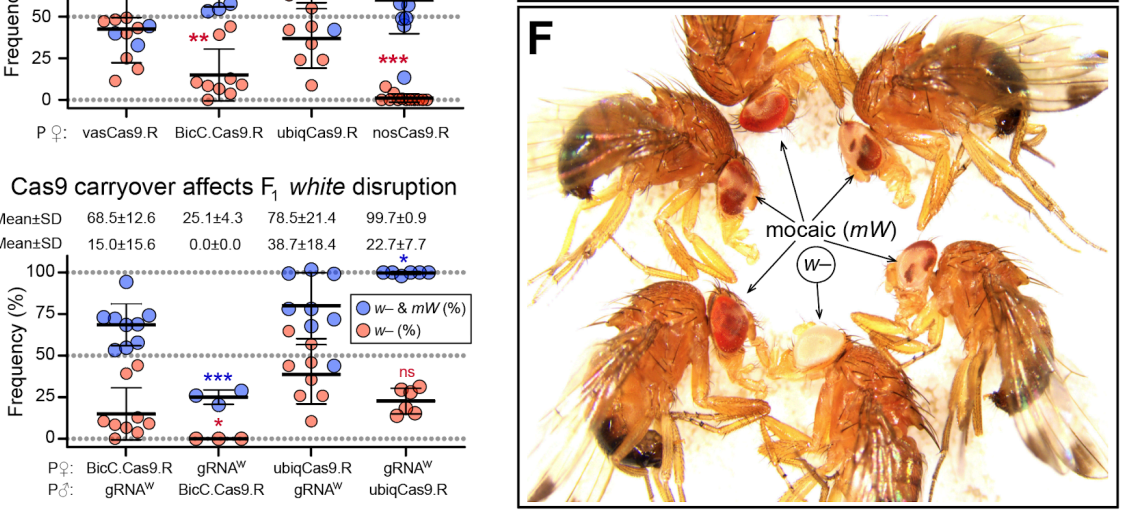

Figure 2. Disruption of yellow and white loci in $F_{1}$ trans-heterozygous flies. (A) The schematic of a genetic cross between Cas 9 and $g R N A^{y}$ flies. To generate $F_{1}$ trans-heterozygous flies, homozygous Cas 9 o (red marker) crossed to homozygous $g R N A^{y}{ }^{\lambda}$ (green marker). The targeted wildtype (wt) yellow gene $(y+$ alleles, yellow stripes on $\mathrm{X}$ chromosome $)$ is on the $\mathrm{X}$ chromosome. Yellow colored thoraxes in $\mathrm{F}_{1}$ flies indicate disruption of the yellow gene ( $y$-). (B) Dot plot depicting the results of disruption of the yellow gene in somatic cells from $\mathrm{F}_{1}$ trans-heterozygous (Cas9/+; $\left.g R N A^{y /+}\right)$ progeny using the Cas9.R strains inherited maternally. (C) Images of $F_{1} y$ - trans-heterozygous and $w t y++$. (D) The schematic of a genetic cross with maternal homozygous Cas9 (red marker) and paternal homozygous gRNAw (purple marker). The white gene is on the $\mathrm{X}$ chromosome ( $w+$ alleles, white stripes on $\mathrm{X}$ chromosome). Red and white eye coloration in the $\mathrm{F}_{1}$ flies indicate somatic disruption of the white gene $(\mathrm{mW})$. (E) Dot plot depicting the results of disruption of the white gene in somatic cells from $\mathrm{F}_{1}$ trans-heterozygous (Cas $\left.9 /+; g R N A^{w /+}\right)$ progeny using the Cas9.R strains inherited maternally. $(\mathbf{F})$ Images of $F_{1}$ trans-heterozygous mosaic white disruption ( $\mathrm{mW}$ ) and complete disruption ( $w-$ ) phenotypes. $(\mathbf{G})$ The schematic of a genetic cross between paternal Cas 9 and maternal gRNA ${ }^{w}$ flies. To generate $F_{1}$ trans-heterozygous flies, homozygous Cas $9{ }^{\lambda}$ (red marker) crossed to homozygous $g R N A^{w}+$ (purple marker). The white gene is on the $\mathrm{X}$ chromosome ( $\mathrm{w}+$ alleles, white stripes). (H) Dot plot depicting the results of disruption of the white gene in somatic cells from $\mathrm{F}_{1}$ transheterozygous (Cas9/+; gRNA $A^{y /+}$ ) progeny using the Cas9.R strains inherited paternally. Plots show the mean \pm SD over at least three biological replicates. Statistical significance was estimated using a two-sided Student's $t$ test with unequal variance. ( $p \geq 0.05^{\text {ns }}, p<0.05^{*}, p<0.01^{* *}$, and $p<0.001^{* * *}$ ). 

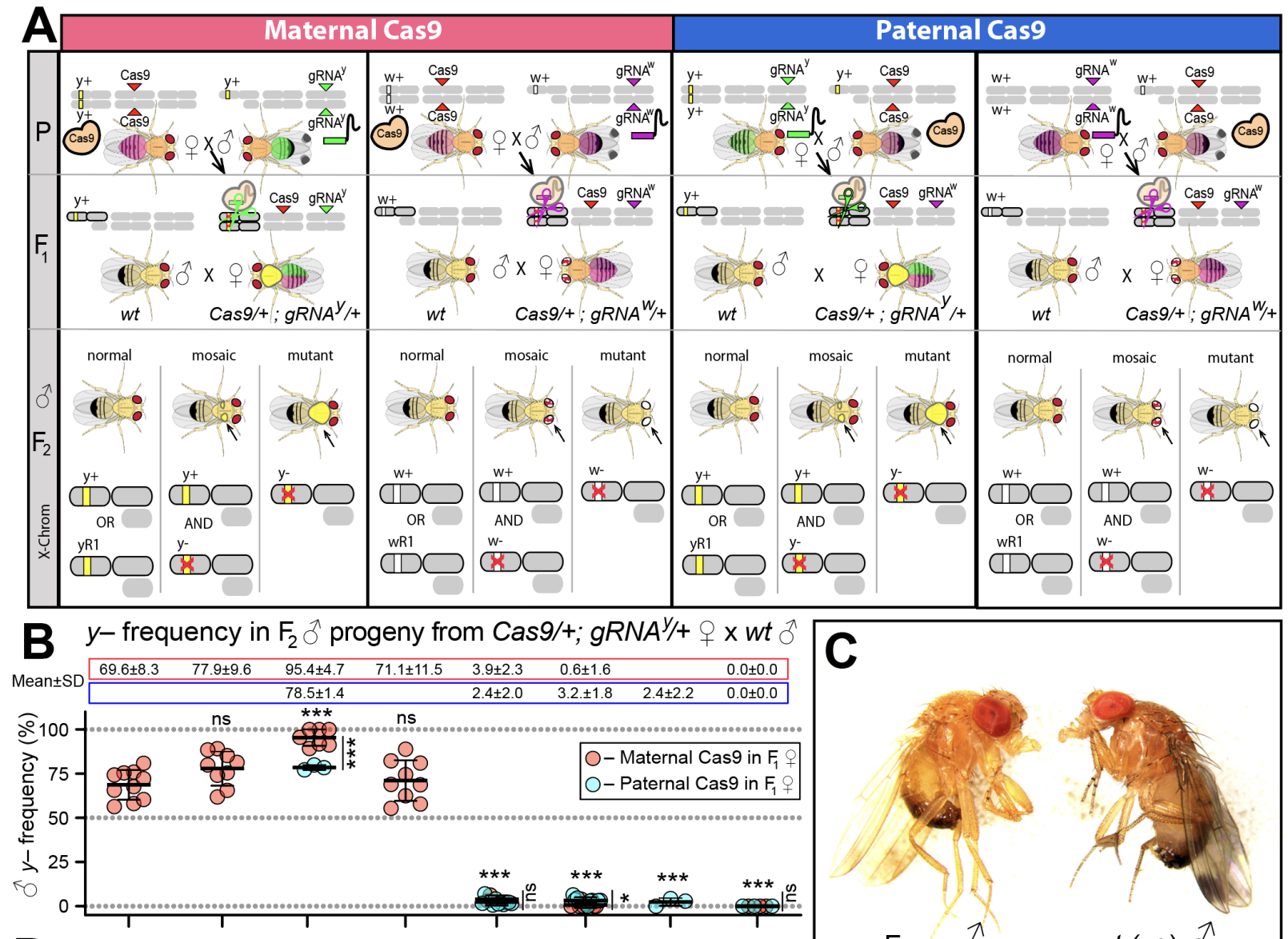

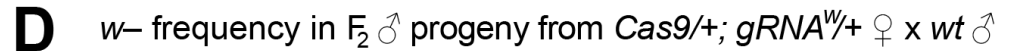
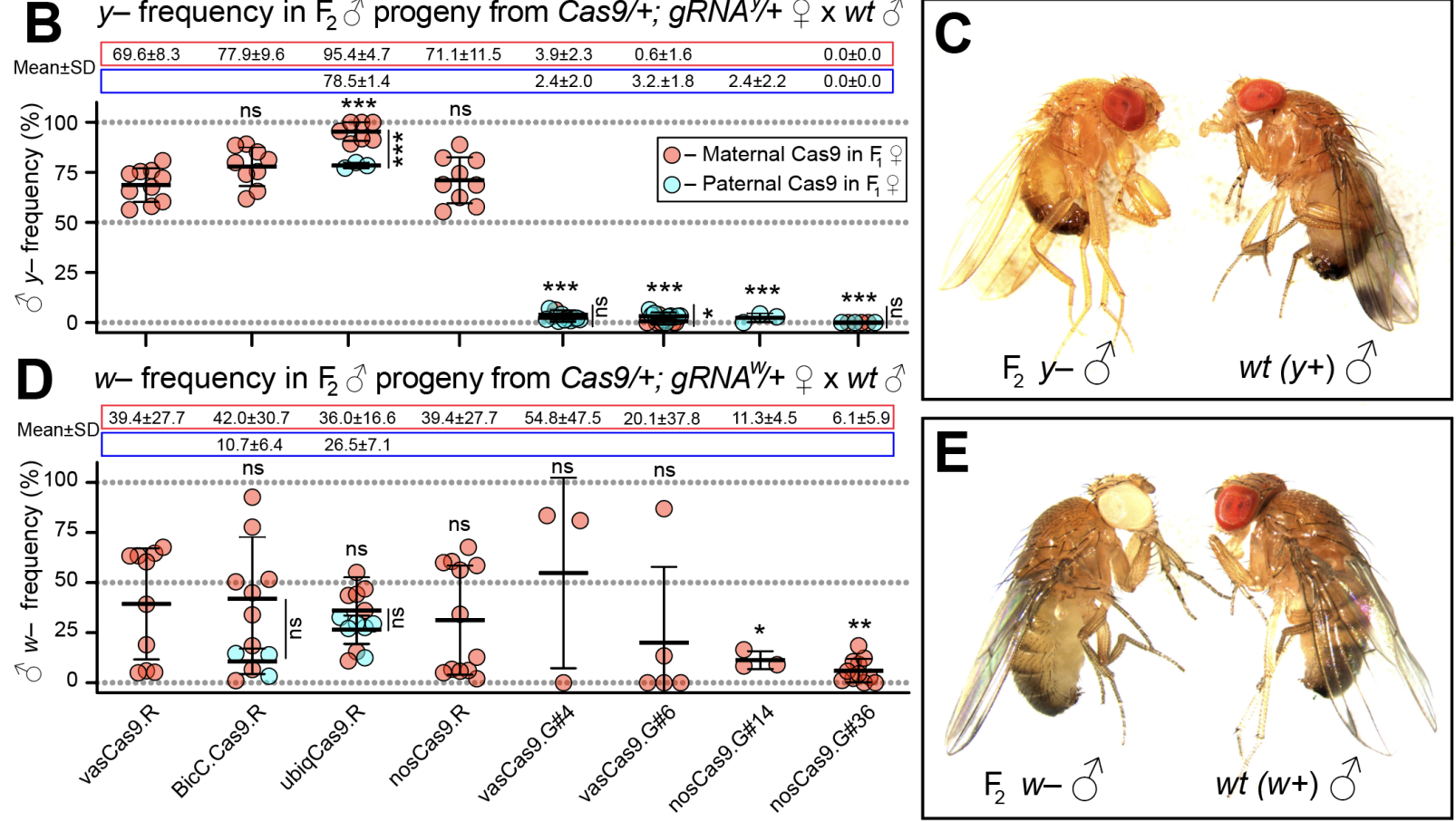

Figure 3. The efficiency of heritable yellow and white mutations. (A) To assess for heritable yellow or white mutations in germ cells, $\mathrm{F}_{1}$ trans-heterozygous Cas9/+; gRNA/+ ${ }^{\prime}$ 's were crossed to wt ${ }^{\text {th' }}$, and yellow or white phenotype was scored in the generated $F_{2}{ }^{\lambda}$ which inherited their mother's $X$ chromosome. Two types of trans-heterozygous of's were used: the $F_{1}$ ○'s harboring maternal Cas 9 (red points), and the $\mathrm{F} 1$ ' 's harboring paternal Cas9 (blue points in B and D). (B) Dot plot depicting the $y$ - frequency in the $F_{2}$ స’'s derived from crosses with the 8 homozygous Cas9 lines generated in this study. (C) Images of the generated $\mathrm{F}_{2} y$ - and $w t(y+) \partial^{\lambda}$. (D) Dot plot depicting the $w$ - frequency in the $\mathrm{F}_{2} \delta^{\lambda}$ derived from crosses with the 8 homozygous Cas9 lines generated in this study. (E) Images of the $F_{2} w-$ and $w t(w+){ }^{\lambda}$. Plots show the mean \pm SD over at least three biological replicates. Statistical significance was estimated using a two-sided Student's $t$ test with equal variance. $\left(p \geq 0.05^{\text {ns }}, p<0.05^{\star}, p<0.01^{* *}\right.$, and $\left.p<0.001^{* * *}\right)$. 

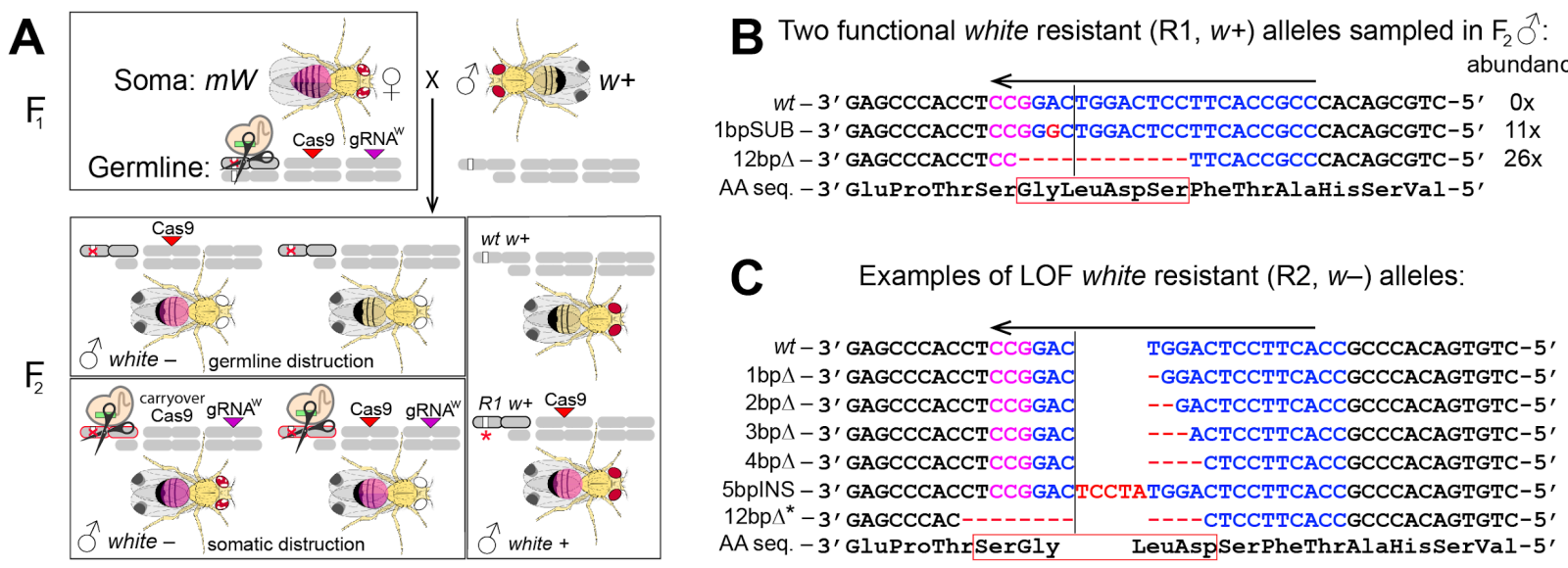

Figure 4. Functional and LOF resistant alleles at the white target loci. (A) Schematic depicting potential $\delta$ progeny from the genetic cross of $\mathrm{F}_{1}$ trans-heterozygous Cas $9 /+; g R N A^{w} /+q$ and $w t(w+){ }^{\lambda}$. The $\mathrm{F}_{1}$ o has mosaic eye coloration $(\mathrm{mW})$ indicating disruption of the white gene in its somatic tissues. Some $w+$ alleles can be mutated in its germ cells resulting in $\mathrm{F}_{2}$ ô harboring the loss-of-function (LOF) $w$ - allele (a crossed out white bar on the X chromosome with a black outline). Some $w+$ alleles can be mutated in the somatic tissues of the $\mathrm{F}_{2} \delta$ that inherited the $g R N A^{w}$ transgene and have the carryover Cas 9 protein (maternally deposited) or Cas 9 transgene (a crossed out white bar on the $\mathrm{X}$ chromosome with a red outline) also resulting in a LOF $w$ - phenotype in $\mathrm{F}_{2} \delta^{\lambda}$. The $\mathrm{F}_{2} \delta^{\hat{t}}$ with normal eye coloration harbors the $w t w+$ allele that escaped cleavage or the functional resistant alleles $(R 1, w+)$ that were mutated and became resistant to cutting by Cas9/gRNA ${ }^{\mathrm{w}}$ but preserved the function of the white gene (a white bar with a red star on the $X$ chromosome with a black outline). Note that LOF $w$-alleles contain insertions or deletions (indels) at the ligated cut site and are also likely resistant to Cas $9 / \mathrm{gRNA}{ }^{\mathrm{w}}$, therefore they are referred to as R2 alleles. To explore the course of high variabilities in the white knockout, we sequenced the white target in $\mathrm{F}_{2} w+\lambda$. (B) Each genotyped $\delta$ from $37 \mathrm{~F}_{2} w+\hat{\delta}$ sampled from multiple independent crosses with each Cas $9 . R$ line had either one-base substitution (1spSUB) or twelve-base deletion $(12 \mathrm{bp} \Delta$ ) directly at the white target sequence. The two R1 alleles persisted for two generations of cutting by Cas $/ \mathrm{gRNA} \mathrm{w}^{\mathrm{w}}$, and maintained $w t$ function of the white gene. No $w t w+$ alleles were identified in any sampled $F_{2} w+{ }^{*}$. (C) LOS R2 allele sampled in $\mathrm{F}_{2} w-\hat{\delta}$. Note that the two sampled R2 alleles are in-frame ( $3 \mathrm{bp} \Delta$ and $\left.12 \mathrm{bp} \Delta^{*}\right)$, and yet they cannot restore the $w t$ function. The sequence alignment of induced LOF alleles against the wt reference white sequence (58). The 20 bases of gRNAw (in blue) and its PAM (purple) are depicted over the white target sequence. Arrows point the direction of the gRNA ${ }^{w}$ target. Mutated bases (red letters) and/or their absence (red dashes) are indicated relative to the wt sequence. 
D. suzukii homozygous yellow-knockout strains generated in the study:

$$
\begin{aligned}
& w t \text { (reference) - 3' GGTCGTCTGTGGGTCCTGGACACCGGA A|CCGTGGGAATTGGCAAC-5 } \\
& \text { NC \& CA wt strains - 3' GGTCGTCTGTGGGTCCTGGACACCGGA ACCGTGGGCATTGGCAAC-5' } \\
& \text { 1bpINS - 3' GGTCGTCTGTGGGTCCTGGACACCGGACA_CCGTGGGCATTGGCAAC-5' } \\
& \text { 6bp } \Delta \text { - 3' GGTCGTCTGTGGGTTCTGGACA---- - CCGTGGGCATTGGCAAC-5' } \\
& \text { 6bp } \Delta \text { - 3' GGTCGTCTGTGGGTCCTGGACA----- -CCGTGGGCATTGGCAAC-5' } \\
& \text { 2bpINS \& 1bp } \triangle-3^{\prime} \text { GGTCGTCTGTGGGTCCTGGACACCGGACTCCGTGGGCATTGGCAAC-5' } \\
& \text { 5bpINS \& 4bp } \Delta-3^{\prime} \text { GGTCGTCTGTGGGTCCTGGACACCGGATGTATTGGGCATTGGCAAC-5' } \\
& 1 \mathrm{bp} \Delta \text { - 3' GGTCGTCTGTGGGTTCTGGACACCGGA -CCGTGGGCATTGGCAAC-5 } \\
& \text { AA seq. (wt) - 3' GlyArgLeuTrpValLeuAspThrGly ThrValGlyIleGlyAsn-5' } \\
& \text { AA seq. (6bp } \Delta)-3^{\prime} \text { GlyArgLeuTrpValLeuAspThrGly ThrValGlyIleGlyAsn-5' }
\end{aligned}
$$

Figure 5. SWD yellow knockout (LOF) strains. We established eight independent homozygous viable $y-$ knockout strains. The sequence alignment of induced LOF alleles against the wt reference yellow sequence (58). The 20 bases of gRNA are in blue, while the PAM sequence is in purple. The arrow points the direction of gRNA target. We identified two silent SNPs (grey colored base) in the SWD NC and CA wt stains relative to the wt reference strain (58): one at the 4th gRNA ${ }^{y}$ base and at the 2nd base after PAM silent the yellow targets. Mutated bases (red letters) and/or their absence (red dashes) are indicated relative to the wt sequence. The same six-base-deletion $(6 \mathrm{bp} \Delta$ ) was independently induced in four strains \#2, \#3, \#4, and \#6. The $6 \mathrm{bp} \Delta$ deletes two amino acids (Glycine and Threonine) that results in the LOF $y$ - phenotype (Figs. 2C, 3B).

\section{Supporting Information}

Table S1. Yellow disruption in F1 and F2 progeny (Cas9 $x$ gRNA data).

Table S2. White disruption in F1 and F2 progeny (Cas9 $x$ gRNA data).

Table S3. Cas 9 protein carryover by BicC.Cas $9 . R$ of the white locus. 


\section{References}

1. D. B. Walsh, et al., Drosophila suzukii (Diptera: Drosophilidae): Invasive Pest of Ripening Soft Fruit Expanding its Geographic Range and Damage Potential. Journal of Integrated Pest Management 2, G1-G7 (2011).

2. A. Cini, C. loriatti, G. Anfora, A review of the invasion of Drosophila suzukii in Europe and a draft research agenda for integrated pest management. Bull. Insectology 54, 149-160 (2012).

3. O. Rota-Stabelli, M. Blaxter, G. Anfora, Drosophila suzukii. Curr. Biol. 23, R8-9 (2013).

4. A. B. Langille, E. M. Arteca, J. A. Newman, The impacts of climate change on the abundance and distribution of the Spotted Wing Drosophila (Drosophila suzukii) in the United States and Canada. PeerJ 5, e3192 (2017).

5. J. C. Lee, et al., Infestation of Wild and Ornamental Noncrop Fruits by Drosophila suzukii (Diptera: Drosophilidae). Annals of the Entomological Society of America 108, 117-129 (2015).

6. D. Mazzi, E. Bravin, M. Meraner, R. Finger, S. Kuske, Economic Impact of the Introduction and Establishment of Drosophila suzukii on Sweet Cherry Production in Switzerland. Insects 8 (2017).

7. D. A. Yeh, F. A. Drummond, M. I. Gómez, X. Fan, The Economic Impacts and Management of Spotted Wing Drosophila (Drosophila Suzukii): The Case of Wild Blueberries in Maine. J. Econ. Entomol. 113, 1262-1269 (2020).

8. M. P. Bolda, R. E. Goodhue, F. G. Zalom, Spotted Wing Drosophila: Potential Economic Impact of a Newly Established Pest. ARE Update 13, 5-8 (2010).

9. G. De Ros, S. Conci, T. Pantezzi, G. Savini, The economic impact of invasive pest Drosophila suzukii on berry production in the Province of Trento, Italy. Journal of Berry Research 5, 89-96 (2015).

10. L. A. Dos Santos, et al., Global potential distribution of Drosophila suzukii (Diptera, Drosophilidae). PLoS One 12, e0174318 (2017).

11. ,Season-long programs for control of Drosophila suzukii in southeastern U.S. blueberries. Crop Prot. 81, 76-84 (2016).

12. D. R. Haviland, E. H. Beers, Chemical Control Programs for Drosophila suzukii that Comply With International Limitations on Pesticide Residues for Exported Sweet Cherries. J Integr Pest Manag 3, F1-F6 (2012).

13. ,Control of spotted wing drosophila, Drosophila suzukii, by specific insecticides and by conventional and organic crop protection programs. Crop Prot. 54, 126-133 (2013).

14. F. R. M. Garcia, Drosophila suzukii Management (Springer Nature).

15. N. Desneux, A. Decourtye, J.-M. Delpuech, The sublethal effects of pesticides on beneficial arthropods. Annu. Rev. Entomol. 52, 81-106 (2007). 
16. F. G. Z. Brian E Gress, Identification and risk assessment of spinosad resistance in a California population of Drosophila suzukii. Pest Management Science 75, 1270-1276 (2019).

17. E. F. Knipling, Possibilities of Insect Control or Eradication Through the Use of Sexually Sterile Males. J. Econ. Entomol. 48, 459-462 (1955).

18. A. P. Krüger, et al., Radiation effects on Drosophila suzukii (Diptera: Drosophilidae) reproductive behaviour. J. Appl. Entomol. 143, 88-94 (2019).

19. G. Lanouette, et al., The sterile insect technique for the management of the spotted wing drosophila, Drosophila suzukii: Establishing the optimum irradiation dose. PLoS One 12, e0180821 (2017).

20. H. Laven, A possible model for speciation by cytoplasmic isolation in the Culex pipiens complex. Bull. World Health Organ. 37, 263-266 (1967).

21. L. O'Connor, et al., Open release of male mosquitoes infected with a wolbachia biopesticide: field performance and infection containment. PLoS Negl. Trop. Dis. 6, e1797 (2012).

22. J. Engelstädter, A. Telschow, Cytoplasmic incompatibility and host population structure. Heredity 103, 196-207 (2009).

23. J. Cattel, et al., Back and forth Wolbachia transfers reveal efficient strains to control spotted wing drosophila populations. J. Appl. Ecol. 55, 2408-2418 (2018).

24. K. Nikolouli, F. Sassù, L. Mouton, C. Stauffer, K. Bourtzis, Combining sterile and incompatible insect techniques for the population suppression of. J. Pest Sci. 93, 647-661 (2020).

25. A. Buchman, J. M. Marshall, D. Ostrovski, T. Yang, O. S. Akbari, Synthetically engineered gene drive system in the worldwide crop pest. Proc. Natl. Acad. Sci. U. S. A. 115, 47254730 (2018).

26. F.-C. Chu, W. Klobasa, N. Grubbs, M. D. Lorenzen, Development and use of a piggyBacbased jumpstarter system in Drosophila suzukii. Arch. Insect Biochem. Physiol. 97 (2018).

27. M. F. Schetelig, Y. Yan, Y. Zhao, A. M. Handler, Genomic targeting by recombinasemediated cassette exchange in the spotted wing drosophila, Drosophila suzukii. Insect Mol. Biol. 28, 187-195 (2019).

28. H. M. M. Ahmed, F. Heese, E. A. Wimmer, Improvement on the genetic engineering of an invasive agricultural pest insect, the cherry vinegar fly, Drosophila suzukii. BMC Genet. 21, 139 (2020).

29. M. Paris, et al., Near-chromosome level genome assembly of the fruit pest Drosophila suzukii using long-read sequencing. Sci. Rep. 10, 11227 (2020).

30. P. Kalajdzic, M. F. Schetelig, CRISPR/Cas-mediated gene editing using purified protein in Drosophila suzukii. Entomol. Exp. Appl. 164, 350-362 (2017).

31. F. Li, M. J. Scott, CRISPR/Cas9-mediated mutagenesis of the white and Sex lethal loci in 
the invasive pest, Drosophila suzukii. Biochem. Biophys. Res. Commun. 469, 911-916 (2016).

32. S. Kondo, R. Ueda, Highly improved gene targeting by germline-specific Cas 9 expression in Drosophila. Genetics 195, 715-721 (2013).

33. X. Ren, et al., Optimized gene editing technology for Drosophila melanogaster using germ line-specific Cas9. Proc. Natl. Acad. Sci. U. S. A. 110, 19012-19017 (2013).

34. S. J. Gratz, et al., Genome engineering of Drosophila with the CRISPR RNA-guided Cas9 nuclease. Genetics 194, 1029-1035 (2013).

35. Z. Yu, et al., Highly efficient genome modifications mediated by CRISPR/Cas9 in Drosophila. Genetics 195, 289-291 (2013).

36. O. Kanca, et al., An efficient CRISPR-based strategy to insert small and large fragments of DNA using short homology arms. Elife 8 (2019).

37. D. Li-Kroeger, et al., An expanded toolkit for gene tagging based on MiMIC and scarless CRISPR tagging in. Elife 7 (2018).

38. N. P. Kandul, et al., Transforming insect population control with precision guided sterile males with demonstration in flies. Nat. Commun. 10, 84 (2019).

39. M. Li, et al., Eliminating Mosquitoes with Precision Guided Sterile Males. bioRxiv, 2021.03.05.434167 (2021).

40. J. Champer, A. Buchman, O. S. Akbari, Cheating evolution: engineering gene drives to manipulate the fate of wild populations. Nat. Rev. Genet. 17, 146-159 (2016).

41. K. M. Esvelt, A. L. Smidler, F. Catteruccia, G. M. Church, Concerning RNA-guided gene drives for the alteration of wild populations. Elife 3 (2014).

42. M. Li, et al., Germline Cas9 expression yields highly efficient genome engineering in a major worldwide disease vector, Aedes aegypti. Proc. Natl. Acad. Sci. U. S. A. 114, E10540-E10549 (2017).

43. H. Sano, A. Nakamura, S. Kobayashi, Identification of a transcriptional regulatory region for germline-specific expression of vasa gene in Drosophila melanogaster. Mech. Dev. 112, 129-139 (2002).

44. M. Van Doren, A. L. Williamson, R. Lehmann, Regulation of zygotic gene expression in Drosophila primordial germ cells. Curr. Biol. 8, 243-246 (1998).

45. N. P. Kandul, et al., Assessment of a Split Homing Based Gene Drive for Efficient Knockout of Multiple Genes. G3 (2019) https:/doi.org/10.1534/g3.119.400985.

46. O. S. Akbari, D. Oliver, K. Eyer, C.-Y. Pai, An Entry/Gateway cloning system for general expression of genes with molecular tags in Drosophila melanogaster. BMC Cell Biol. 10, 8 (2009).

47. M. M. van Oers, J. M. Vlak, H. O. Voorma, A. A. M. Thomas, Role of the 3' untranslated region of baculovirus p10 mRNA in high-level expression of foreign genes. J. Gen. Virol. 80 
( Pt 8), 2253-2262 (1999).

48. B. D. Pfeiffer, J. W. Truman, G. M. Rubin, Using translational enhancers to increase transgene expression in Drosophila. Proc. Natl. Acad. Sci. U. S. A. 109, 6626-6631 (2012).

49. F. Port, H.-M. Chen, T. Lee, S. L. Bullock, Optimized CRISPR/Cas tools for efficient germline and somatic genome engineering in Drosophila. Proc. Natl. Acad. Sci. U. S. A. 111, E2967-76 (2014).

50. R. Lehmann, C. Nüsslein-Volhard, The maternal gene nanos has a central role in posterior pattern formation of the Drosophila embryo. Development 112, 679-691 (1991).

51. A. H. Sturtevant, Experiments on sex recognition and the problem of sexual selection in Drosophila (1915) https:/doi.org/10.1037/h0074109 (March 11, 2021).

52. N. P. Kandul, J. Liu, J. B. Bennett, J. M. Marshall, O. S. Akbari, A confinable home and rescue gene drive for population modification. Elife 10 (2021).

53. G. Terradas, et al., Inherently confinable split-drive systems in Drosophila. Nat. Commun. 12, 1480 (2021).

54. D. G. Gibson, et al., Enzymatic assembly of DNA molecules up to several hundred kilobases. Nat. Methods 6, 343-345 (2009).

55. L. A. Baena-Lopez, C. Alexandre, A. Mitchell, L. Pasakarnis, J.-P. Vincent, Accelerated homologous recombination and subsequent genome modification in Drosophila. Development 140, 4818-4825 (2013).

56. M. F. Schetelig, X. Nirmala, A. M. Handler, Pro-apoptotic cell death genes, hid and reaper, from the tephritid pest species, Anastrepha suspensa. Apoptosis 16, 759-768 (2011).

57. C. Concha, et al., Efficient germ-line transformation of the economically important pest species Lucilia cuprina and Lucilia sericata (Diptera, Calliphoridae). Insect Biochem. Mol. Biol. 41, 70-75 (2011).

58. J. C. Chiu, et al., Genome of Drosophila suzukii, the spotted wing drosophila. G3 3, 22572271 (2013).

59. A. R. Bassett, C. Tibbit, C. P. Ponting, J.-L. Liu, Highly Efficient Targeted Mutagenesis of Drosophila with the CRISPR/Cas9 System. Cell Rep. 6, 1178-1179 (2014).

60. X. Li, J. C. Heinrich, M. J. Scott, piggyBac-mediated transposition in Drosophila melanogaster: an evaluation of the use of constitutive promoters to control transposase gene expression. Insect Mol. Biol. 10, 447-455 (2001).

61. P. Mali, et al., RNA-guided human genome engineering via Cas9. Science 339, 823-826 (2013). 